\title{
Phosphaturic mesenchymal tumor in the oral cavity causing oncogenic osteomalacia: A case report
}

\author{
Sarafidou Anastasia*, Karkos D Petros, Tsinaslanidou Zinovia and Constantinidis Jannis \\ Department of Otolaryngology-Head Neck Surgery, AHEPA University Hospital, Aristotle University of Thessaloniki, Greece
}

\begin{abstract}
Background: Phosphaturic Mesenchymal Tumor (PMT) is a rare type of mesenchymal neoplasm that is associated with oncogenic osteomalacia, resulting in serious musculoskeletal problems. Since it exhibits rare occurrence and random possible siting, diagnosis is often delayed for years.

Methods: We report a case of a patient diagnosed with a small PMT in the oral cavity, who remained undiagnosed for months. The combination of PET/CT scan and systemic venous sampling of fibroblast growth factor 23 , that is secreted from the tumor, confirmed the diagnosis.

Results: Total resection of the tumor was uncomplicated and totally curative.

Conclusions: Hence, physicians should stay vigilant and have in mind the characteristics of PMTs, in order to include them in the differential diagnosis of osteomalacia.
\end{abstract}

\section{Introduction}

Phosphaturic mesenchymal tumors (PMTs) are very rare neoplasms, which are usually associated with Tumor Induced Osteomalacia (TIO). TIO is a paraneoplastic syndrome related to renal phosphate wasting. The tumor cells produce a peptide hormone-like substance known as fibroblast growth factor 23 (FGF23), which regulates phosphate levels. Specifically, FGF23 prevents reabsorption and increases the excretion of phosphate in the renal proximal tubules. In addition, FGF23 inhibits 1-a-hydroxylase, that reduces levels of 1, 25-dihydroxy vitamin D3. Thus, overexpression of FGF23 by the tumor leads to increased clearance of phosphate in the urine, mobilization of phosphate and calcium from the bones, and reduction of osteoblastic activity, resulting in impaired bone mineralization and progressive bony tissue loss [1]. Since first being described, only a few case series of PMTs and less than 500 single cases reports have been published in the literature.

Phosphaturic mesenchymal tumors can arise either from bone or soft tissue [2]. They are characterized by unpredictable localization. The most common location of the tumors involves the extremities (95\%), following by head and neck area (5\%). Most of the PMTs in the head and neck sites are located in the sinonasal cavity, whilst the intraoral location is the second most common [3-5].

PMTs affects both genders almost equally. They are more often diagnosed in middle-aged adults, although the age of the patients can range from 3 to 73 years [5-7]. Nevertheless, PMTs among elderly patients are relatively rare [8].

Clinical manifestations of PMTs include bone and joint pain, multiple pathological bone fractures, osteopenia, gradual muscular weakness, and atrophy, resulting in mobility impairment. These symptoms are nonspecific, and many patients remain asymptomatic for years, leading to a challenging and difficult diagnostic process. The final diagnosis is commonly delayed for years due to the rare occurrence of the tumors, their relatively slow growth and lack of clinical suspicion.
According to literature, there is an estimated delay to the detection of the primary tumor of over 5 years on average $[9,10]$. Typical laboratory findings include hypophosphatemia, normocalcemia, normal or low levels of 1, 25-dihydroxy vitamin D3 and elevated levels of FGF23. These findings should arise significant suspicions of PMTs.

The majority of these tumors are benign and histological evidence of malignancy is rare [5]. Nevertheless, in malignant ceses, it has been reported that there is metastatic potential [10].

The majority of the tumors are usually misdiagnosed, because of their small size and their unpredictable location. However, it is crucial, yet challenging, to localize the tumors associated with oncogenic osteomalacia. Surgical resection of the tumor is the gold standard treatment, since complete resection with negative margins is curative.

In this article we present an intraoral case of PMT with the typical characteristics of tumor induced osteomalacia.

\section{Case report}

A 48-year-old woman was referred to the rheumatologists with a 2-year-old history of progressive weakness of lower limbs, multiple atraumatic fractures of the pelvic bones bilaterally and the right humerus bone, resulting in total hip replacement. She had significant mobility problems and ended up using a walker. She was experiencing

${ }^{*}$ Correspondence to: Sarafidou Anastasia, Department of Otolaryngology-Head Neck Surgery, AHEPA University Hospital, Aristotle University of Thessaloniki, Greece, Tel: +30 6936974477; E-mail: tesisaraf@gmail.com

Key words: phosphaturic mesenchymal tumor, osteomalacia, fibroblast growth factor

Received: August 12, 2020; Accepted: September 01, 2020; Published: September 04, 2020 
severe bone pain not responding well to NSAIDs and simple analgesics. Her medical and family history was unremarkable for hereditary bone diseases and other conditions, as well as, negative for tobacco and alcohol use. Laboratory results revealed severe hypophosphatemia, hyperphosphaturia with normal renal function, normal calcium and parathyroid hormone levels, and low levels of 1,25-dihydroxy vitamin D3. Multiple myeloma work up was also negative, as well as for any autosomal diseases. After several months of diagnostic turmoil, these findings raised the suspicion of oncogenic osteomalacia. Testing for FGF23, which performed in a specialized medical center in USA, showed an increased serum level $(838 \mathrm{RU} / \mathrm{ml})$ of the phosphatonin and confirmed the diagnosis of tumor-induced osteomalacia. The patient was treated with NSAIDs, high doses of phosphate and vitamin-D supplements and underwent multiple physiotherapy sessions with poor response. Given the high clinical suspicion of PMT, a whole-body PET-CT scan was performed which showed homogenous radiotracer uptake by an intraoral lesion in the right mandible (Figure 1). There was no observable pattern of other lesions. A complete head and neck and intraoral examination was conducted revealing a red fixed and firm mass in the right mandible with no associated neck lymphadenopathy (Figure 2).

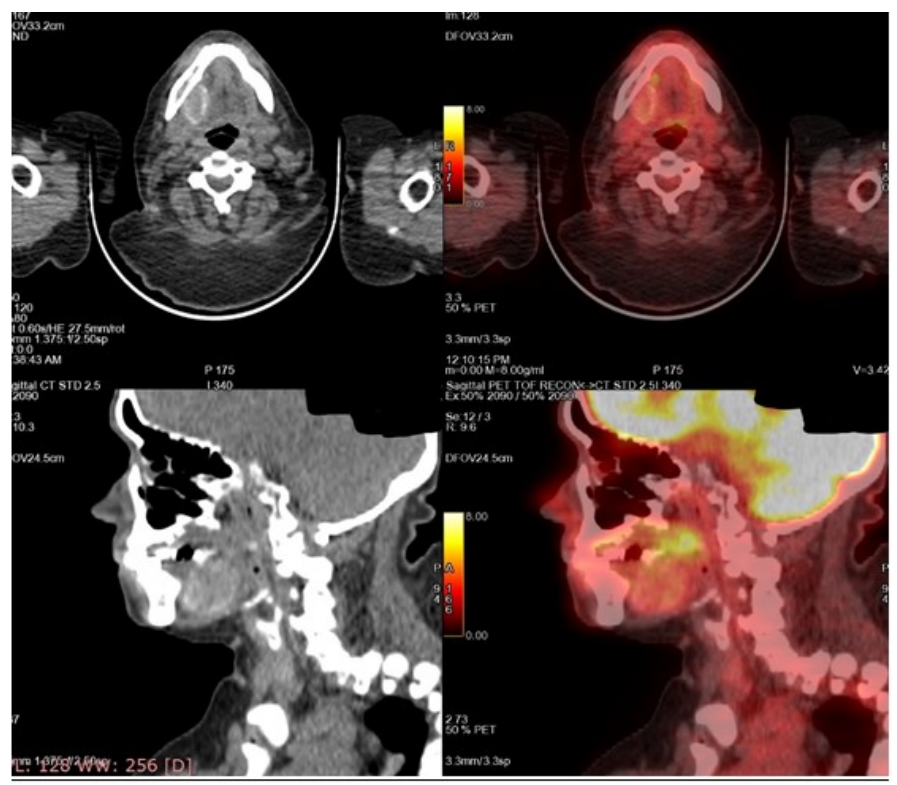

Figure 1. PET-CT indicated the high uptake lesion in the oral cavity

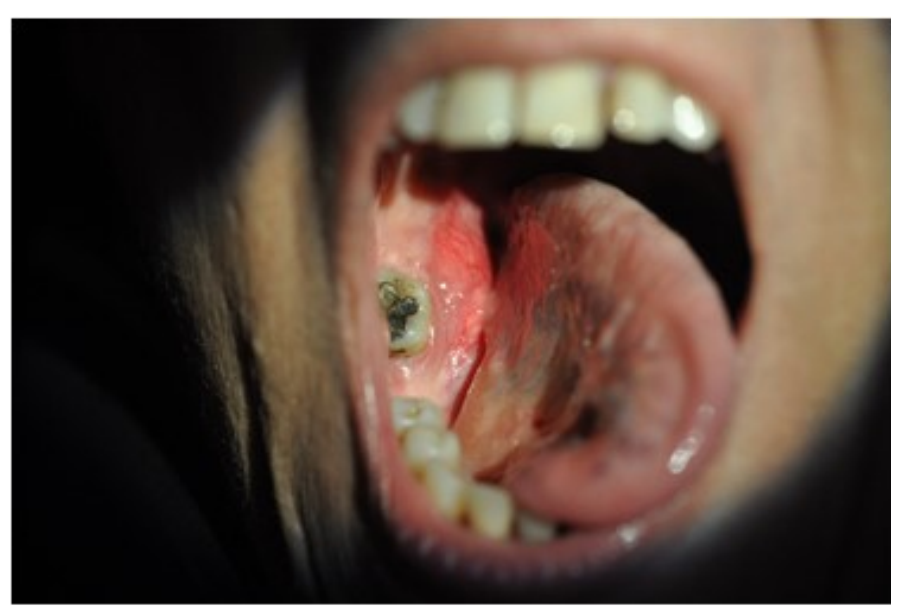

Figure 2. Phosphaturic mesenchymal tumor in the oral cavity

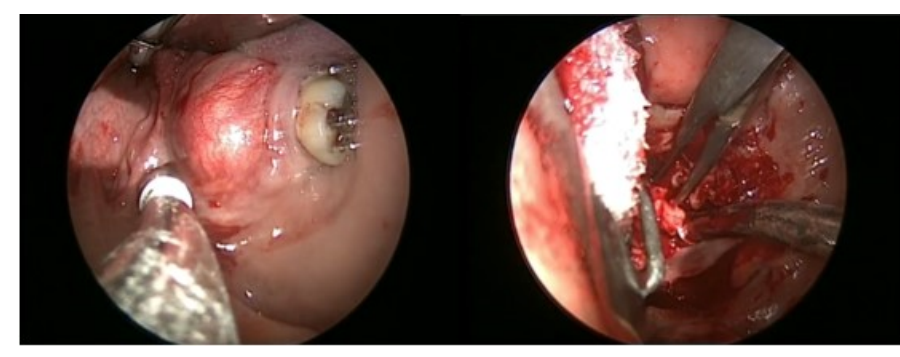

Figure 3. Tumor resection was performed intraorally

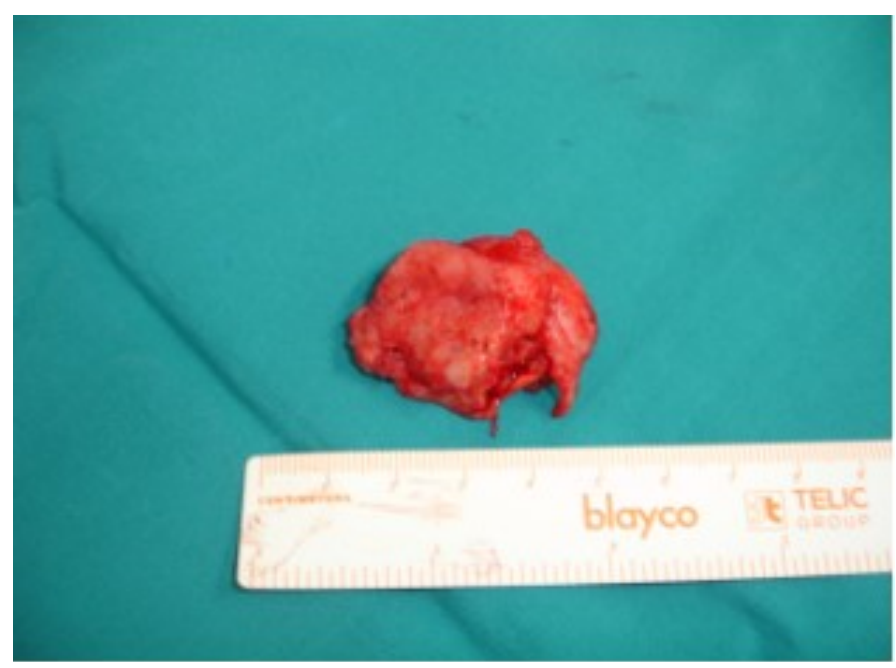

Figure 4. Tumor after complete resection

Subsequent MRI and CT scan confirmed a 41X34X17 mm mass located in the floor of the oral cavity in contact with the right mandible. After detailed discussion and appropriate consent, it was determined that the best plan of action would be to proceed with surgery. Complete surgical resection of the tumor was performed intraorally under general anesthesia and resected completely (Figures 3 and 4). Histopathological analysis confirmed the diagnosis of a phosphaturic mesenchymal tumor mixed connective tissue type.

Postoperatively, the patient's blood phosphorus levels gradually returned to normal within a few days and the patient's symptoms decreased dramatically, resulting in resolution of osteomalacia. Additionally, the lady reported a significant improvement in pain and range of movement and an annual postoperative follow up MRI scan was clear

\section{Discussion'}

PMTs are a rare entity of soft tissue tumors that are characterized by excessive release of phosphatonins, especially FGF23. This overproduction of FGF23 increases renal excretion of phosphate, decreases reabsorption of phosphate and calcium and, also, interferes in the metabolic process of 1,25-dihydroxycholecalciferol vitamin D3. These pathological mechanisms lead to tumor-induced osteomalacia, with the characteristic presenting symptoms of bone pain, muscular weakness, multiple bone fractures, myopathies etc. [7,11,12].

The first clinical presentation of PMTs was described in 1947 by McCance, while in 1959 was the first association of tumors and osteomalacia by Prader [5]. The term PMT was introduced by Weidner and Cruz in 1987, when they suggested dividing the tumors in four histological subtypes: phosphaturic mesenchymal tumor mixed connective tissue type which occurs in soft tissue, osteoblastoma like, 
ossifying fibroma type and non-ossifying fibroma type, which occurs in bone [13]. Meanwhile, over $90 \%$ of mesenchymal tumor-induced osteomalacia cases are attributed to PMT/ MCTs (Mixed Connective Tissue type) [14].

Even though a few cases of PMTs have been reported in literature, it is paramount to consider this diagnosis in all patients with hypophosphatemic osteomalacia. The main biochemical findings of this disorder, that should arise suspicion of the diagnosis of PMT, include, besides hypophosphatemia, low serum calcitriol concentrations, high serum alkaline phosphatase and high serum FGF23 concentrations [15]. FGF23 has been described as a useful common diagnostic factor for tumor induced osteomalacia [16,17]. Due to its rarity, the purpose of this paper is to report a new case of PMT located intraorally, and the clinical, laboratory, pathological characteristics where typical, as reported in such cases in literature worldwide.

The localization of PMTs in the head and neck area is quite rare (5\%) $[3,4]$. Moreover, it is estimated that there has been an underreport of PMT cases, especially in the head and neck area. So far, it takes 2,5 years to recognize oncogenic osteomalacia after the onset of clinical symptoms [17] and on average 5 years to locate the responsible neoplasm $[9,10]$. The main problem with PMTs are their nonspecific musculoskeletal symptoms, their commonly small size and that they can be located in any part of the human body, making their identification a challenging process [18]. Consequently, a stepwise approach starting with a thorough medical history and physical examination of the patient, as well as, selective venous sampling, followed by functional imaging, and eventually anatomical imaging, is crucial.

Complete and definitive surgical excision with wide margins is the first-choice treatment. As shown in our case, complete resection of the tumor is possible and therapeutic, resulting in absolute reversal of the symptoms and biochemical abnormalities. Prognosis of most PMT cases is exceptional, reflecting the commonly benign behavior of the tumors. However, clinical behavior of PMTs cannot always be predicted based on histological, clinical, and radiological features, therefore, long-term follow-up of the cases is essential. Moreover, it must be underlined that further research in the genetic and diagnostic approach of this disorder is essential and is expected to shed some light on this "atypically" presenting tumor. crucial to be done [19-24].

\section{Conclusion}

Phosphaturic mesenchymal tumors leading to tumor induced osteomalacia should be included in the differential diagnosis of patients with nonspecific predominant musculoskeletal problems. Early identification and localization of the causative neoplasm is of great importance since it could cure the problem and improve quality of life by limiting years of pain, discomfort, and possible disabilities of the patient.

\section{References}

1. Ghorbani-Aghbolaghi A, Darrow MA, Wang T (2017) Phosphaturic mesenchymal tumor (PMT): Exceptionally rare disease, yet crucial not to miss. Autopsy Case Reports 7: 32-37.

2. Hautmann AH, Hautmann MG, Kölbl O, Herr W, Fleck M (2015) Tumor-Induced Osteomalacia: an Up-to-Date Review. Curr Rheumatol Rep 17: 512. [Crossref]

3. Deep NL, Cain RB, McCullough AE, Hoxworth JM, Lal D (2014) Sinonasal phosphaturic mesenchymal tumor: Case report and systematic review. Allergy Rhinol (Providence) 5: 162-167. [Crossref]

4. Gardner KH, Shon W, Folpe AL, Wieland CN, Tebben PJ, Baum CL (2013) Tumorinduced osteomalacia resulting from primary cutaneous phosphaturic mesenchymal tumor: A case and review of the medical literature. J Cutan Pathol 40:780-4; quiz 779. [Crossref]
5. Qari H, Hamao-Sakamoto A, Fuselier C, Cheng YSL, Kessler H, et al. (2016) Phosphaturic Mesenchymal Tumor: 2 New Oral Cases and Review of 53 Cases in the Head and Neck. Head Neck Pathol 10: 192-200. [Crossref]

6. Okamiya T, Takahashi K, Kamada H, Hirato J, Motoi T, et al. (2015) Oncogenic osteomalacia caused by an occult paranasal sinus tumor. Auris Nasus Larynx 42: 167-169.

7. Richardson AL, Richardson OK (2019) Phosphaturic mesenchymal tumor: Case report. Radiol Case Reports 14: 1518-1524. [Crossref]

8. Aziz KT, McCarthy EF, Morris CD (2017) Oncogenic osteomalacia secondary to a metastatic phosphaturic mesenchymal tumor in the talus: A case report and review of the literature. JBJS Case Connect 7: e40. [Crossref]

9. Mori Y, Ogasawara T, Motoi T, Shimizu Y, Chikazu D, et al. (2010) Tumor-induced osteomalacia associated with a maxillofacial tumor producing fibroblast growth factor 23: report of a case and review of the literature. Oral Surg Oral Med Oral Pathol Oral Radiol Endod 109: e57-e63. [Crossref]

10. Pelo S, Gasparini G, Garagiola U, D'Amato G, Saponaro G, et al. (2018) Phosphaturic mesenchymal tumor, an unusual localization in head and neck. J Surg Case Rep 2018: rjy091. [Crossref]

11. Chong WH, Molinolo AA, Chen CC, Collins MT (2011) Tumor-induced osteomalacia Endocrine Related Cancer 18: R53-R77.

12. Jan De Beur SM (2005) Tumor-induced osteomalacia. JAMA 294: 1260-1267. [Crossref]

13. Woo VL, Landesberg R, Imel EA, Singer SR, Folpe AL, et al. (2009) Phosphaturic mesenchymal tumor, mixed connective tissue variant, of the mandible: report of a case and review of the literature. Oral Surg Oral Med Oral Pathol Oral Radiol Endod 108: 925-932. [Crossref]

14. Xiao X, Sun X, Ni P, Huang Y, Xie T (2018) Phosphaturic mesenchymal tumor and related wound problem. Medicine (Baltimore) 97: e12507. [Crossref]

15. Alonso G, Varsavsky M (2016) Tumour-induced osteomalacia: An emergent paraneoplastic syndrome. Endocrinol Nutr 63: 181-186. [Crossref]

16. Qiu S, Cao LL, Qiu Y, Yan P, Li ZX, et al. (2017) Malignant phosphaturic mesenchymal tumor with pulmonary metastasis. Medicine (Baltimore) 96: e6750. [Crossref]

17. Lee JY, Park HS, Han S, Lim JK, Hong N, et al. (2017) Localization of oncogenic osteomalacia by systemic venous sampling of fibroblast growth factor 23. Yonsei Med J 58: 981-987. [Crossref]

18. Shiba E, Matsuyama A, Shibuya R, Yabuki K, Harada H, et al. (2016) Immunohistochemical and molecular detection of the expression of FGF23 in phosphaturic mesenchymal tumors including the non-phosphaturic variant. Diagn Pathol 11: 26. [Crossref]

19. Zuo QY, Wang H, Li W, Niu XH, Huang YH, et al. (2017) Treatment and outcomes of tumor-induced osteomalacia associated with phosphaturic mesenchymal tumors: Retrospective review of 12 patients. BMC Musculoskelet Disord 18: 403. [Crossref]

20. Wasserman JK, Purgina B, Lai CK, Gravel D, Mahaffey A, et al. (2016) Phosphaturic Mesenchymal Tumor Involving the Head and Neck: A Report of Five Cases with FGFR1 Fluorescence In Situ Hybridization Analysis. Head Neck Pathol 10: 279-285. [Crossref]

21. Folpe AL (2019) Phosphaturic mesenchymal tumors: A review and update. Semin Diagn Pathol 36: 260-268. [Crossref]

22. Adnan Z, Nikomarov D, Weiler-Sagie M, Roguin Maor N (2019) Phosphaturic mesenchymal tumors among elderly patients: a case report and review of literature. Endocrinol Diabetes Metab Case Rep 2019: 18-0139. [Crossref]

23. Kurien R, Rupa V, Thomas M (2019) Varied presentation of sinonasal phosphaturic mesenchymal tumour: report of a case series with follow-up. Eur Arch Otorhinolaryngol 276: 1677-1684. [Crossref]

24. Takashi Y, Kinoshita Y, Ito N, Taguchi M, Takahashi M, et al. (2017) Tumor-induced osteomalacia caused by a parotid tumor. Intern Med 56: 535-539. [Crossref]

Copyright: (C2020 Sarafidou A. This is an open-access article distributed under the terms of the Creative Commons Attribution License, which permits unrestricted use, distribution, and reproduction in any medium, provided the original author and source are credited. 